\title{
Quantitative proteomics of maize roots treated with a novel protein hydrolysate: Comparative study with transcriptomics highlights the molecular mechanisms responsive to biostimulants
}

Leonard Barnabas Ebinezer ${ }^{1}$, Cinzia Franchin ${ }^{2,3}$, Anna Rita Trentin ${ }^{1}$, Paolo Carletti ${ }^{*}$, Sara Trevisan ${ }^{1}$, Ganesh Kumar Agrawal4,5, Randeep Rakwal4,5,6, Silvia Quaggiotti ${ }^{1}$, Giorgio Arrigoni ${ }^{2,3 *}$, Antonio Masi ${ }^{1}$

\footnotetext{
${ }^{1}$ Department of Agronomy, Food, Natural Resources, Animals, and Environment, University of Padova, Padua, Italy,

${ }^{2}$ Department of Biomedical Sciences, University of Padova, Padua, Italy,

${ }^{3}$ Proteomics Center, University of Padova and Azienda Ospedaliera di Padova, Italy,

${ }^{4}$ Research Laboratory for Biotechnology and Biochemistry (RLABB), Nepal,

${ }^{5}$ GRADE (Global Research Arch for Developing Education) Academy Private Limited, Nepal,

${ }^{6}$ Faculty of Health and Sport Sciences, University of Tsukuba, Tsukuba, Ibaraki, Japan
}

\section{Correspondence:}

Dr. Paolo Carletti

paolo.carletti@unipd.it

Dr. Giorgio Arrigoni

giorgio.arrigoni@unipd.it 


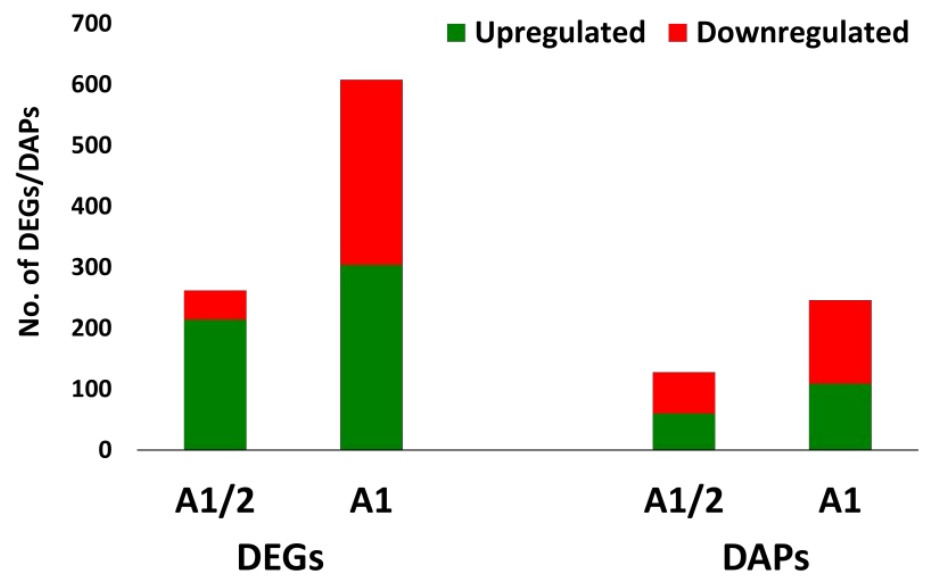

Fig. S1 Number of up and down-regulated DEGs and DAPs identified with the transcriptomic and proteomic analyses 

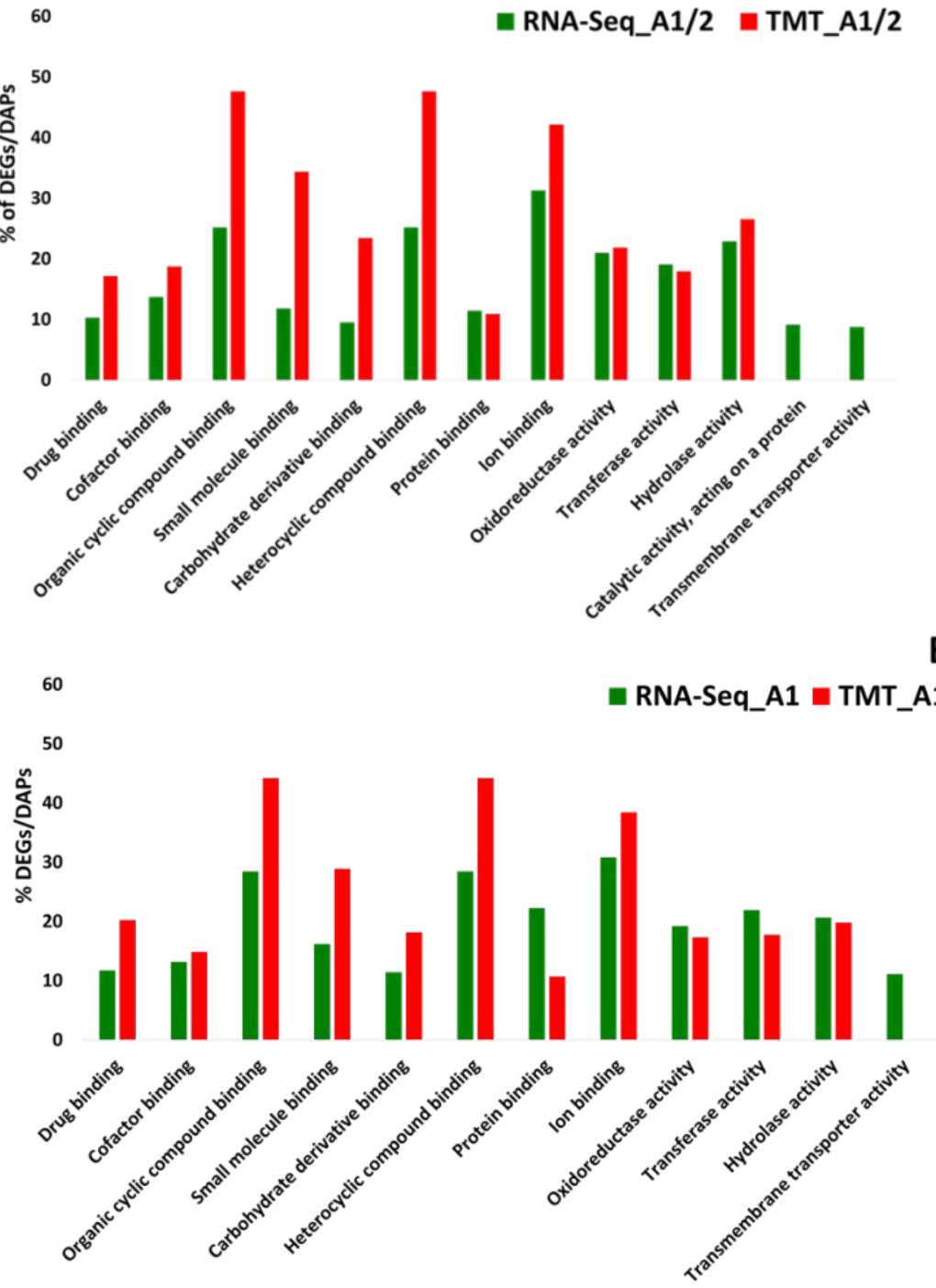

Fig. S2 Classification of DEGs and DAPs based on molecular functions. A and B represent \% of DEGs and DAPs identified with $A P R^{\circledR}$ at $A 1 / 2$ and $A 1$ concentrations, respectively.

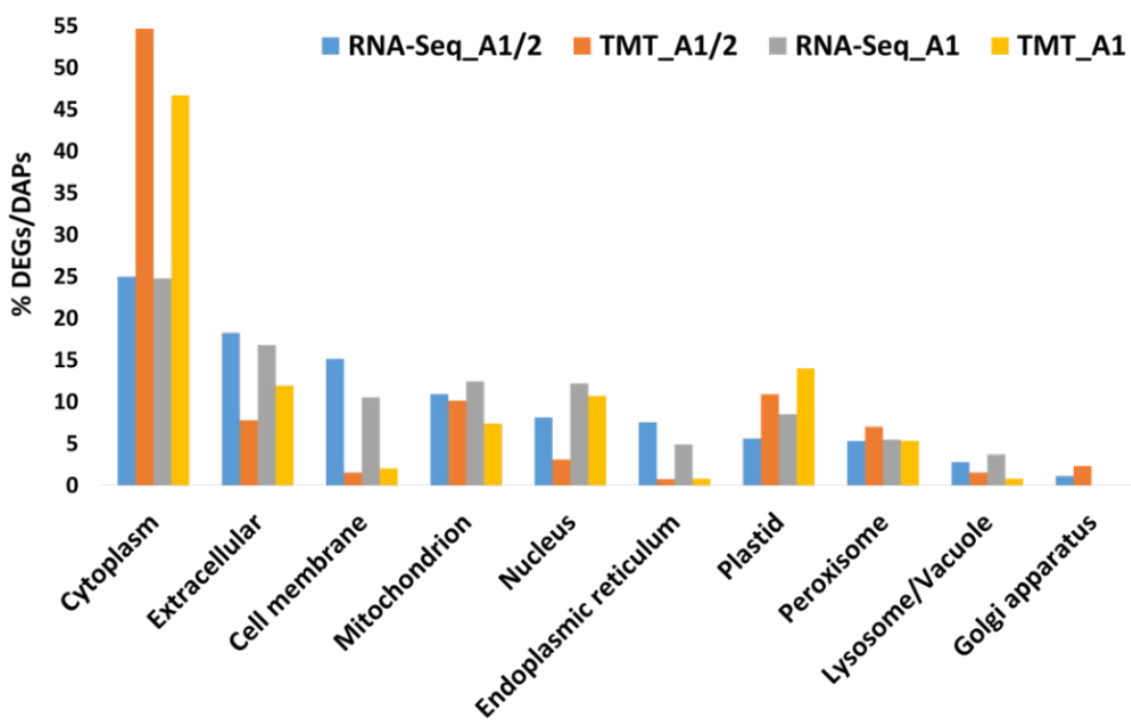

Fig. S3 Sub-cellular localization prediction of DEGs and DAPs. 
Transcriptomics

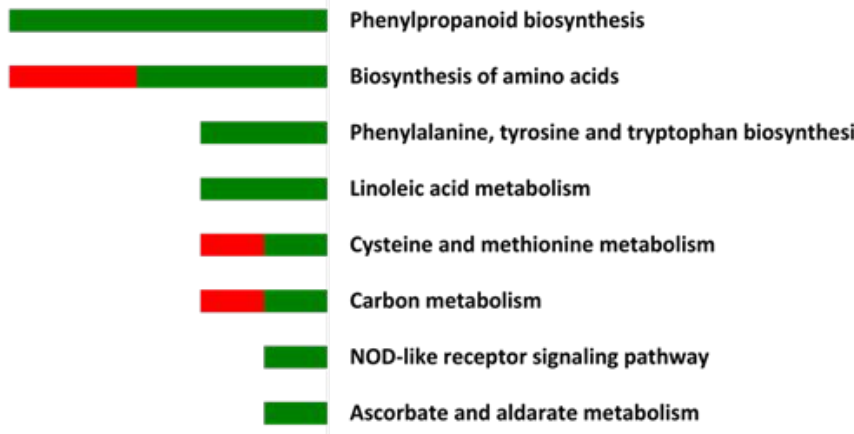

Proteomics

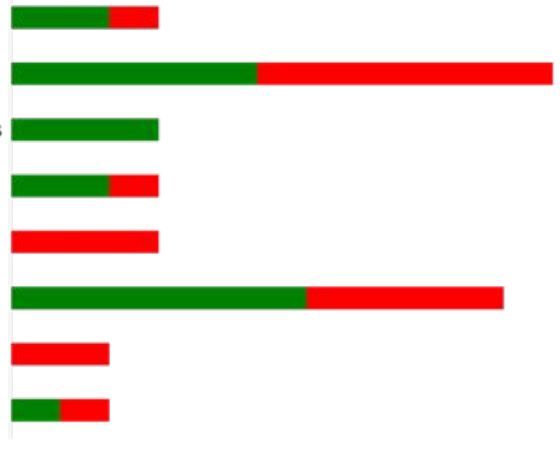
Stilbenoid, diarylheptanoid and gingerol biosynthesis Phenylalanine metabolism Brassinosteroid biosynthesis Plant hormone signal transduction Nitrogen metabolism

Inositol phosphate metabolism Monoterpenoid biosynthesis Ubiquinone and other terpenoid-quinone biosynthesis

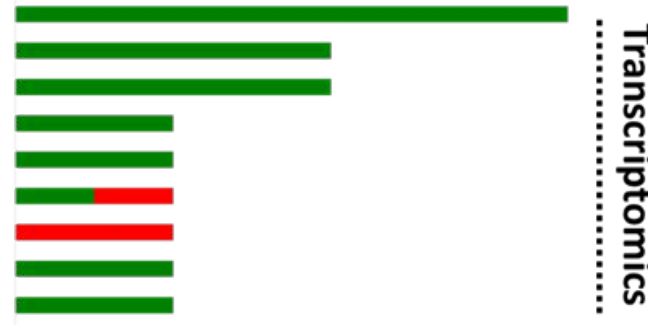

Glyoxylate and dicarboxylate metabolism Glycolysis / Gluconeogenesis Pentose phosphate pathway Glycine, serine and threonine metabolism Starch and sucrose metabolism Drug metabolism - other enzymes Citrate cycle (TCA cycle) Fatty acid metabolism Tryptophan metabolism Pyruvate metabolism Oxidative phosphorylation Peroxisome

Glutathione metabolism AMPK signaling pathway Pyrimidine metabolism Terpenoid backbone biosynthesis Lysine degradation

Propanoate metabolism

Valine, leucine and isoleucine degradation Alanine, aspartate and glutamate metabolism MAPK signaling pathway - plant 2-Oxocarboxylic acid metabolism Carbon fixation in photosynthetic organisms Pentose and glucuronate interconversions Amino sugar and nucleotide sugar metabolism Purine metabolism

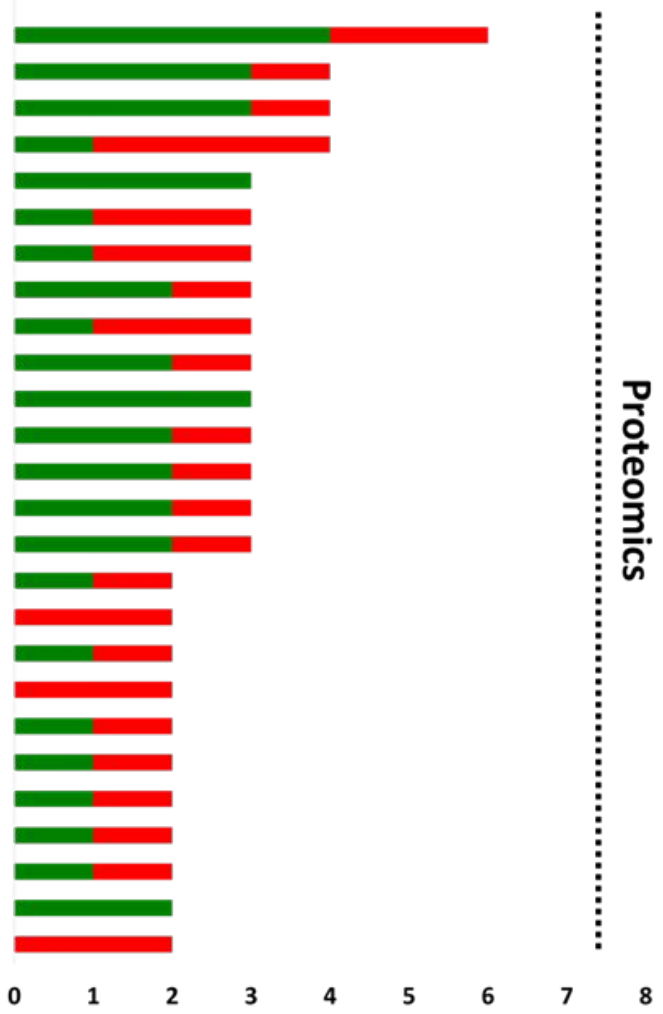

Fig. S4 Common (A) and exclusive (B) pathways regulated at the transcriptome and proteome level with $A 1 / 2 A{ }^{\circledast}{ }^{\circledast}$ treatment. Numbers on the $Y$ axis denote the number of $K O$ accessions mapped with the pathway; Green and red bars indicate up- and down-regulation, respectively. 


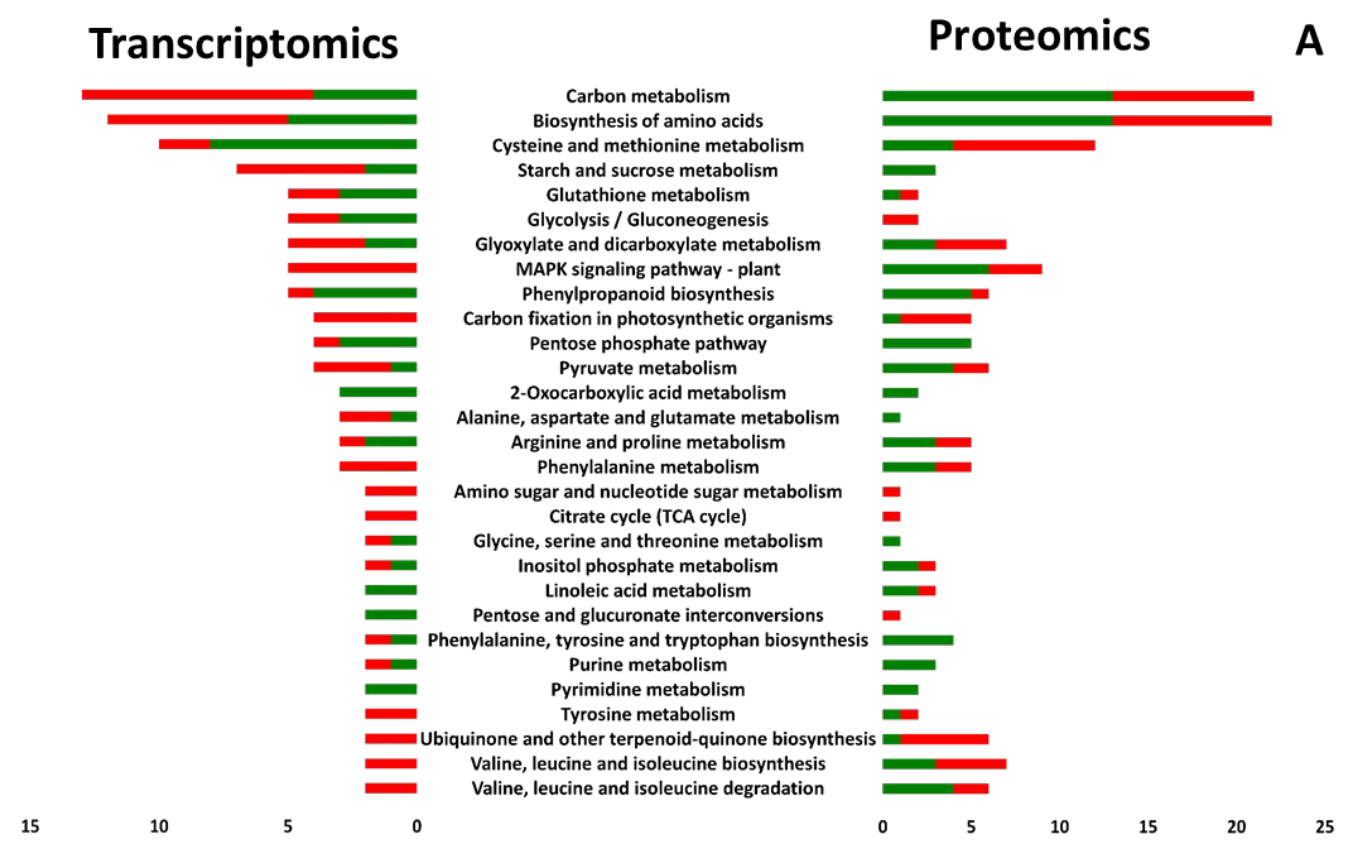

B

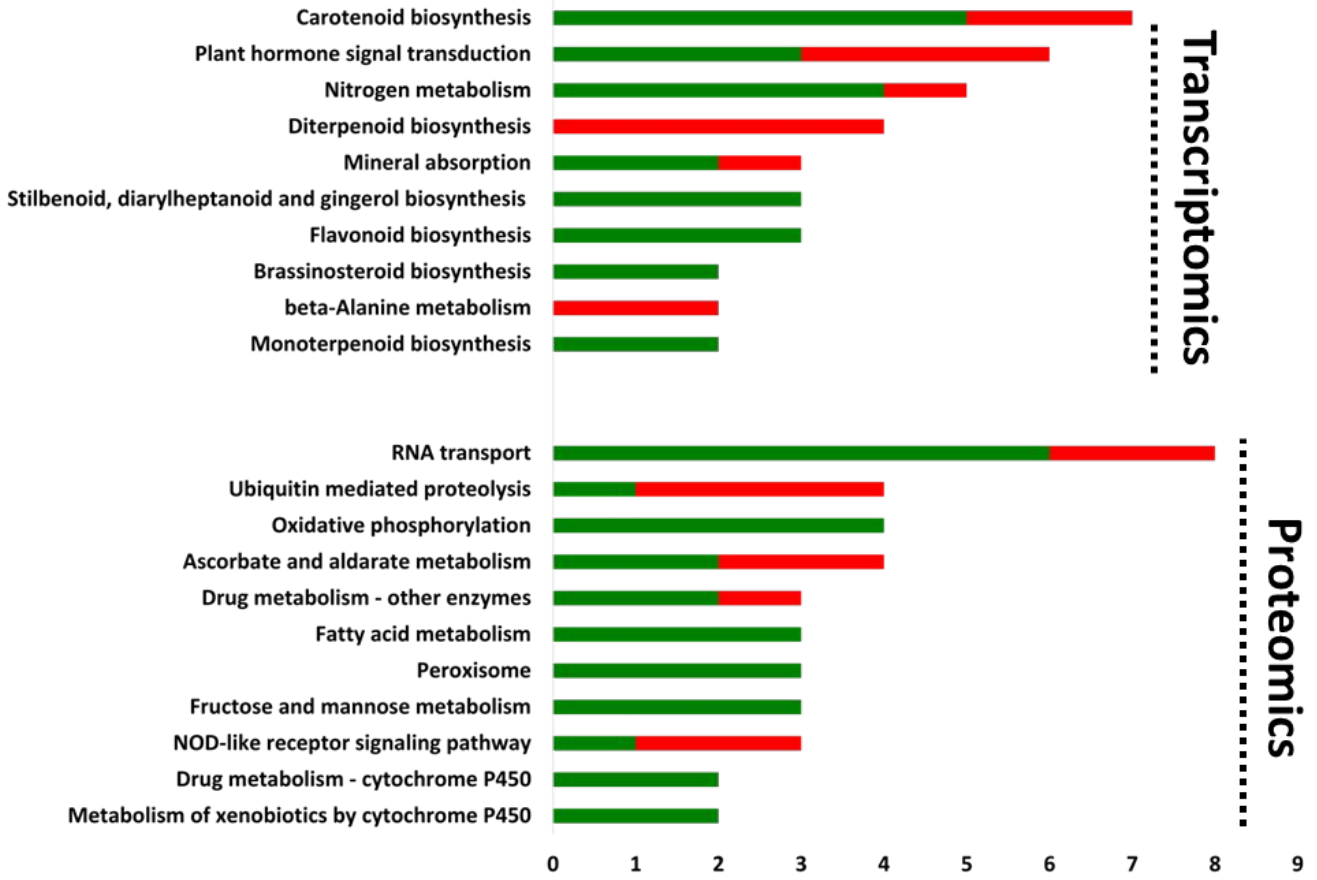

Fig. S5 Common (A) and exclusive (B) pathways regulated at the transcriptome and proteome level with A1 APR ${ }^{\circledR}$ treatment. Numbers on the $Y$ axis denote the number of KO accessions mapped with the pathway; Green and red bars indicate up- and down-regulation, respectively. 